\title{
Un enseignement novateur. Les cours d'Ange Politien à l'université de Florence (1480-1494)
}

The Innovative Teaching of Angelo Poliziano at the University of Florence (1480-1494)

Eine bahnbrechende Lehre. Die Vorlesungen

von Ange Politien an der Universität Florenz (1480-1494)

Una enseñanza novadora. Las clases de Ange Politien

en la universidad de Florencia (1480-1494)

Jean-Marc Mandosio

\section{(2) OpenEdition}

\section{Édition électronique}

URL : https://journals.openedition.org/histoire-education/1830

DOI : $10.4000 /$ histoire-education. 1830

ISSN : 2102-5452

\section{Éditeur}

ENS Éditions

\section{Édition imprimée}

Date de publication : 1 septembre 2008

Pagination : 33-52

ISBN : 978-2-7342-1132-7

ISSN : 0221-6280

Référence électronique

Jean-Marc Mandosio, « Un enseignement novateur. Les cours d'Ange Politien

à l'université de Florence (1480-1494)», Histoire de l'éducation [En ligne], 120 | 2008, mis en ligne le 01 janvier 2013, consulté le 20 mai 2021. URL : http://journals.openedition.org/histoire-education/1830 ; DOI : https://doi.org/10.4000/histoire-education.1830 


\title{
Les cours d'Ange Politien à l'université de Florence (1480-1494)
}

\author{
Jean-Marc MANDOSIO
}

Ange Politien est l'un des humanistes et des poètes les plus célèbres de la fin du Quattrocento. Il est aussi l'un des promoteurs de la méthode philologique moderne, qu'il contribue à développer dans le cadre de ses cours à l'université de Florence, de 1480 à sa mort en 1494. Son parcours universitaire, très bien documenté malgré quelques zones d'ombre ${ }^{1}$, est ici retracé à la lumière de la politique culturelle de Laurent de Médicis et de la stratégie d'autopromotion du jeune professeur, figure de proue, avec Pic de la Mirandole et quelques autres, d'une avant-garde désireuse de renverser l'autorité de la génération précédente. Amateur d'innovations, Politien se singularise constamment : il invente la leçon inaugurale en vers, se sert de l'imprimerie pour faire connaître ses idées, accuse ses collègues d'être des ignorants et finit par remettre en question les distinctions admises entre les disciplines, considérant que le "grammairien " - c'està-dire le philologue - a vocation à traiter tous les domaines de la connaissance. Malgré sa mort précoce, il influence profondément l'humanisme européen.

1 Les deux études principales sur l'enseignement d'Ange Politien et son évolution sont : Vittore Branca, Poliziano e l'umanesimo della parola, Turin, Einaudi, 1983, ch. 5 (" Professore nello Studio fiorentino "), p. 73-90 ; Lucia Cesarini Martinelli, " Poliziano professore allo Studio fiorentino ", dans l'ouvrage collectif La Toscana al tempo di Lorenzo il Magnifico : politica economia cultura arte, Pise, Pacini, 1996, vol. II, p. 463-481. 


\section{I - Le cadre institutionnel}

Le parcours de Politien ne peut s'expliquer sans référence à la situation politique de l'époque. En 1434, le banquier Côme de Médicis (Côme l'Ancien, 1389-1464) prend le pouvoir à Florence en imposant une dictature de fait, tout en maintenant extérieurement les institutions républicaines : "roi sans couronne ", le prince affecte de n'être que "le premier citoyen parmi ses pairs " (civis primus inter pares). À sa mort, son fils Pierre le Goutteux (1416-1469) lui succède, remplacé à son tour par son fils Laurent le Magnifique (1449-1492), que François Guichardin appelait "le plus aimable des tyrans" ${ }^{2}$. Le fils de Laurent, Pierre, dit le Malchanceux ou l'Infortuné (1472-1503), sera renversé en novembre 1494 par une insurrection républicaine consécutive à la " descente " en Italie des troupes du roi de France Charles VIII.

Laurent, qui accède aux affaires en décembre 1469, "refonde " en décembre 1472 l'université (studium generale) de Florence après un long dépérissement. Depuis les années 1450, l'enseignement était donné à des élèves triés sur le volet, dans un cadre non institutionnel (une villa de Careggi appartenant aux Médicis), par les membres de l'Académie florentine, fondée par Côme l'Ancien à l'instigation du philosophe néoplatonicien Marsile Ficin (1433-1499). La nouvelle université est pourvue d'un conseil (gli Ufficiali dello Studio), qui décide en principe de tout : nominations, programmes, salaires. Son siège principal est installé à Pise, qui faisait alors partie de l'État de Florence. L'histoire de cette université devient mouvementée à partir de l'insurrection de 1494 : elle déménage à Prato, avant de cesser ses activités en 1503, lors de la chute de la république de Florence.

Grâce aux registres qui ont été conservés, nous connaissons les noms, les fonctions et les salaires des différents professeurs, nous possédons la liste des étudiants, des livres, bref tous les détails permettant de reconstituer l'histoire de l'université de Florence pendant les trente années de son existence ${ }^{3}$.

2 Cité par Pierre Antonetti, Les Médicis, Paris, Presses universitaires de France, 1997, p. 31. Voir également Ivan Cloulas, Laurent le Magnifique, Paris, Fayard, 1982.

3 Voir Armando Verde, Lo Studio fiorentino (1473-1503) : ricerche e documenti, Florence, Olschki, 1973-1994 (4 vol.). Toutes les informations relatives aux contrats et aux salaires que l'on trouvera plus loin sont tirées de ce monumental ouvrage. 
Les disciplines enseignées sont, dans l'ordre de la progression du cursus $^{4}$ :

- la grammaire (latine), enseignée aux enfants par des grammatici ou " mâ̂tres d'école " (maestri di scuola) ;

- la poétique et la rhétorique, la logique, la philosophie ;

- la médecine, le droit civil, le droit canon, la théologie.

Ainsi, le studium generale tient lieu à la fois, selon nos critères actuels, d'école primaire, de collège et d'université.

Les cours de poétique et rhétorique (poesia et arte oratoria) ont lieu en partie à Pise, en partie à Florence. Ils sont généralement assurés par quatre professeurs, dont le statut et le salaire varient considérablement ${ }^{5}$. Les deux professeurs qui enseignent à Pise touchent un salaire moindre que leurs collègues, plus prestigieux, qui enseignent à Florence. Parmi ces quatre professeurs, il y a presque toujours un Grec - Byzantin émigré - ayant pour mission d'enseigner la langue et la philosophie helléniques ${ }^{6}$. Tous les cours consistent en commentaires de textes (lectiones). Les Grecs faisaient leurs cours en latin, comme l'attestent les leçons inaugurales d'Argyropoulos qui ont été conservées ${ }^{7}$ et les notes prises par son élève Donato Acciaiuoli ${ }^{8}$; les étudiants ne maîtrisaient pas assez le grec, sauf exception, pour qu'il en allât autrement.

Par rapport à l'enseignement délivré dans les trois facultés supérieures (médecine, droit, théologie), dont la structure est fixée de façon presque immuable, la chaire de "poétique et rhétorique " a un caractère relativement expérimental et ouvert. Les professeurs y jouissent d'une latitude beaucoup plus grande que leurs collègues théologiens, juristes ou médecins, astreints à des programmes très stricts, où les thèmes, les textes à étudier et la progression de l'enseignement sont spécifiés dans le détail : tel professeur doit enseigner telle

4 Ibid., vol. I, p. 296 sqq. (où les disciplines sont énumérées dans l'ordre inverse, en partant de la faculté la plus élevée - la théologie - pour terminer par la grammaire).

5 Les enseignants étant liés à l'université de Florence par des contrats le plus souvent annuels, leur position et leur salaire sont réexaminés au terme de chaque année universitaire.

6 Andronic Calliste (mort en 1478), Jean Argyropoulos (1415-1487), Démétrius Chalcondylas (1424-1511) et Jean Lascaris (1445-1534) occupent successivement ce poste. Leurs compétences étant plus rares, les professeurs grecs sont généralement bien rémunérés.

$7 \quad$ Præfationes Johanni Argyropuli dum Florentiæ doceret philosophiam, in Reden und Briefe Italienischer Humanisten : ein Beitrag zur Geschichte der Pädagogik des Humanismus..., éd. Karl Müllner, Vienne, Hölder, 1899.

8 Virginia Brown, "Johannes Argyropoulos, On the Agent Intellect: an edition of ms. Magliabechi V 42 (ff. 224-228v) ", in Essays in honour of Anton Charles Pegis, Toronto, Pontifical Institute of Medieval Studies, 1974, p. 160-175. 
partie du programme à tel moment de l'année, dans telles conditions précises. La poétique et la rhétorique, domaines d'élection des humanistes - en particulier florentins -, n'ont pas cette dimension rigoureusement scolastique et sont enseignées comme des disciplines " modernes".

Le professeur de poétique et rhétorique le plus renommé, jusqu'à l'arrivée d'Ange Politien, est l'humaniste Cristoforo Landino (1424-1498). Il a été nommé dès la fondation de l'université, en 1473, pour enseigner à Florence même, et non à Pise. Il avait alors quarante-neuf ans et était au sommet de sa gloire : auteur d'ouvrages érudits en latin (Disputationes Camaldulenses) et en langue vulgaire (commentaire de la Divine comédie), il est chancelier de la Seigneurie de Florence, poste très honorifique traditionnellement occupé par un grand lettré. Il sera rétribué jusqu'à sa mort - à l'âge de soixante-quatorze ans -, mais sera dispensé d'enseignement (immunis) durant les deux dernières années. Son salaire de professeur, qui n'a jamais varié, est de 300 florins. En comparaison, si l'on prend l'exemple de l'année 1473-74, ses trois collègues touchent respectivement 80 florins (Lorenzo da Colle, Pise), 165 florins (Bartolomeo da Prato Vecchio, Pise) et 200 florins (Andronic Calliste, Florence).

\section{II - Une carrière fulgurante}

Telle est la situation lorsque Agnolo Ambrogini, dit Ange Politien, est nommé professeur de "poétique et rhétorique " à l'âge de vingt-cinq ans, le 29 mai 1480.

Né le 14 juillet 1454, orphelin de père, il est issu d'une famille de la petite bourgeoisie de Montepulciano (Mons Politianus). Très tôt remarqué pour son intelligence, il bénéficie, en compagnie du jeune Laurent de Médicis (qui a cinq ans de plus que lui), des cours donnés par Ficin, Landino, Argyropoulos, etc., à l'Académie de Careggi. Dans sa jeunesse, il brille par ses dons poétiques, en latin comme en grec et en langue vulgaire, et jouit rapidement d'une réputation de grande érudition. En 1473, son ami Laurent de Médicis, devenu son prince, le prend à son service en tant que secrétaire particulier et, deux ans plus tard, le nomme précepteur de ses fils Pierre (né en 1472, qui lui succédera) et Jean (futur pape Léon X, né en 1475). Ange Politien, jeune homme désargenté, a besoin de cette promotion sociale, mais il est d'un caractère ombrageux et supporte difficilement sa situation ambiguë d'ami/employé, d'autant plus que l'épouse de Laurent, Clarice, lui est hostile : elle n'apprécie guère son esprit 
caustique et ses vues anticonformistes concernant l'éducation des enfants ${ }^{9}$. Écarté de ses fonctions de précepteur, il quitte Florence en 1479. Il accepte de revenir l'année suivante, sur les instances de Laurent, mais à la condition que son indépendance financière soit désormais garantie. Laurent suggère donc au conseil de l'université de le nommer professeur de poétique et rhétorique, ce qui équivaut à un ordre.

La nomination d'Ange Politien, imposée par Laurent de Médicis, n’a pas seulement pour but de régler un problème personnel. Elle est clairement perçue par le conseil de l'université comme un coup de force visant à introduire un très jeune homme, l'un des représentants les plus en vue de la nouvelle génération des humanistes, dans une institution fondée moins de dix ans auparavant mais qui n'est sans doute pas aussi dynamique que le souhaiterait Laurent, comme le montre l'autorité encore incontestée de Landino, âgé de cinquante-six ans et dont l'enseignement commence à paraître démodé. Le relatif conservatisme du conseil de l'université est ainsi mis en cause ; le premier souci dudit conseil sera par conséquent de s'assurer que le nouveau venu ne bouleversera pas de fond en comble les valeurs établies, et notamment qu'il n'entreprendra pas de supplanter Landino.

Les contrats d'Ange Politien ${ }^{10}$ spécifient d'entrée de jeu que ses cours doivent avoir lieu "à une autre heure que ceux de maître Landino ", " afin qu'on ne puisse dire ni tacitement ni explicitement qu'il est en concurrence avec ledit maître " ${ }^{11}$. Il est également astreint à " la condition et obligation de tenir publiquement à l'université de Florence, en plus de ses autres cours, un cours en langue grecque, soit de poétique, soit de rhétorique ", et d'" enseigner régulièrement chez lui, de façon privée, les Erotemata ${ }^{12}$ et les éléments et rudiments

9 Dans les œuvres complètes d'Ange Politien, publiées en 1498, figure un court essai visant à démontrer " que la colère chez les enfants est souvent le signe d'un excellent tempérament " (Guod ira in pueris optimæ sæpe indolis est argumentum).

10 Son dossier individuel est reproduit par Armando Verde, Lo Studio fiorentino, op. cit., vol. II, p. 26-29.

11 ... alia hora quam dominus Christophorus Landinus, contrat pour l'année 1480-81; ... alia hora quam dominus Christophorus Landinus, ita ut nec tacite nec expresse dici potest ipsum esse concurrentem dicti domini Christophori, contrat pour l'année 1482-83.

12 Titre d'un manuel de grec - les Questions - écrit à la fin du XIVe siècle par le Byzantin Manuel Chrysoloras (mort en 1415), réputé être la première grammaire grecque en usage dans l'Europe latine. Un autre ouvrage analogue, portant le même titre, fut publié en 1480 à Milan par Constantin Lascaris. 
des lettres grecques ${ }^{13}$. Cette obligation d'assurer un cours d'initiation à la langue grecque ne durera pas plus de deux ans, semble-t-il.

Nous pouvons mesurer le succès de l'enseignement d'Ange Politien à la progression de sa rémunération. Celle-ci est de 100 florins à ses débuts en 1480-81 et passe à 125 florins dès l'année suivante. La troisième année de son enseignement voit le salaire initial de Politien doubler, puisqu'il est de 200 florins en 1482-83. Nouvelle hausse en 1486-87, Politien recevant dès lors 250 florins pour son enseignement, et encore trois ans plus tard (1489-90), où sa rémunération atteint 300 florins, avant d'être portée à 450 florins en 1491-92.

On voit ainsi qu'en une dizaine d'années à peine, le salaire d'Ange Politien rattrape puis dépasse celui de Landino, fixé dès 1473 à 300 florins. À trentesept ans, en 1491, il est le professeur le mieux payé. À l'autre bout de l'échelle, la rémunération d'autres professeurs de poétique et rhétorique sans grand prestige intellectuel est infime : l'obscur Naldo Naldi, par exemple, ne touche que 50 florins en 1483-84 (quatre fois moins que Politien) ; il passe à 70 florins en 1487 en devenant professeur de grammaire ; son remplaçant, le tout aussi obscur Giorgio Benigni, reçoit 30 florins en 1487-88, 50 florins l'année suivante, et n'obtient qu'en 1491 un salaire de 80 florins, passant ainsi de 12 à 17 \% du salaire de Politien, dont la croissance est démesurée en comparaison.

Le succès d'Ange Politien ne se traduit pas seulement par un afflux d'argent, mais aussi par la liberté de plus en plus grande dont il jouit quant au contenu de son enseignement. À partir de 1488, il obtient le privilège - également accordé à Landino et au Grec Chalcondylas ${ }^{14}$ - de donner " deux leçons le jour qui lui conviendra ${ }^{15}$ dans les disciplines de son choix " ${ }^{16}$, " tant en latin qu'en grec, selon ce qu'il estimera utile aux étudiants " ${ }^{17}$, et à partir de 1491 il lui est concédé de donner " quatre leçons, tant grecques que latines, deux le

13 ... cum hac conditione et obligatione quod dictus dominus Angelus teneatur legere publice in studio Florentino, ultra alias lectiones suas, etiam unam lectionem in græco, sive in poetica sive in oratoria facultate, et privatim domi Herotimata, contrat pour l'année 1481-82 ; ... et cum conditione quod teneatur docere privatim domi Herotimata et elementa et rudimenta græcarum litterarum, contrat pour l'année 1482-83.

14 Entré en 1475 à l'université de Florence avec un salaire de 168 florins, Démétrius Chalcondylas passe à 200 florins en 1478 et à 250 florins en 1489 .

15 La clause de non-concurrence avec Landino est donc devenue caduque.

16 ... duas lectiones quolibet die in iis facultatibus quibus [vult], contrat pour l'année 1488-89.

17 ... cum latine tum græce eas lectiones quas studiosis utiles esse cognoverit, contrat pour l'année 1489-90. 
matin, deux l'après-midi, sur ce qu'il jugera lui-même le plus utile et le plus fructueux pour la jeunesse de Florence ${ }^{18}$.

\section{III - Des débuts fracassants (1480-1481)}

Qu'y avait-il donc de si remarquable dans l'enseignement d'Ange Politien ? Dès sa première année de cours ${ }^{19}$, la volonté de rupture est manifeste : il fait tout pour se distinguer de son collègue et ancien professeur Landino, avec lequel il entre ouvertement en rivalité, confirmant les craintes des autorités universitaires ${ }^{20}$. Landino avait trente ans de plus que lui et représentait la " vieille école " de l'humanisme. Les auteurs favoris de Landino étaient Cicéron et Virgile ; Politien fait son premier cours sur Quintilien et Stace. Cicéron était considéré par la plupart des humanistes antérieurs comme le modèle insurpassable de toute éloquence ; Politien récuse l'hégémonie cicéronienne en présentant à ses élèves l'Institution oratoire de Quintilien et se pose, ce faisant, en continuateur de Laurent Valla (1405-1457), qui avait attiré l'attention sur le contre-modèle que constituait la rhétorique de Quintilien. De même, Virgile était considéré, selon une tradition constante, comme le plus grand des poètes latins ; Politien met en avant une tout autre poétique : celle des Silves de Stace, référence très " moderne ", puisque les œuvres de ce poète du I ${ }^{\text {er }}$ siècle après J.-C. avaient été redécouvertes au début du XV siècle par le Pogge (Poggio Bracciolini, 1380-1459).

En contestant du haut de sa chaire la hiérarchie traditionnelle des auctoritates, Politien se fait le porte-parole d'une " nouvelle vague " humaniste. Le fait qu'il s'agit bien d'un effort concerté, visant à promouvoir la jeune génération avec l'appui de Laurent de Médicis, est confirmé par la lettre adressée en juin 1484 à ce dernier par Jean Pic de la Mirandole (1463-1494) - encore plus jeune que son ami Politien -, qui s'en prend durement aux poésies de

18 ... quatuor lectiones tum græcas tum latinas, duas mane, duas etiam post meridiem, quas ipsemet utiliores et fructuosiores Florentinæ juventutis esse cognoverit, contrat pour l'année 1491-92.

19 Le programme des cours d'Ange Politien, tel qu'il nous est connu, est récapitulé dans le tableau qui figure en annexe.

20 Landino s'en est sans doute ému auprès du conseil de l'université, puisque le contrat de 1482-83 renouvelle avec plus d'insistance l'interdiction faite à Politien de le concurrencer (voir ci-dessus, note 11$)$. 
Dante et de Pétrarque, toujours portées aux nues par les Florentins alors qu'elles sont largement surpassées par les poésies de Laurent lui-même ${ }^{21}$.

Ange Politien conteste aussi la manière dont les auteurs antiques étaient commentés par Landino, qui privilégiait l'interprétation allégorique de style néoplatonicien. Là encore, il s'inscrit dans la lignée de Valla : "Le Politien voulait se présenter à ses nouveaux auditeurs de l'université comme l'héritier et le continuateur de l'orientation grammaticale et antiquaire de lecture des textes " - promue à Rome par Valla et représentée à Venise par Ermolao Barbaro (1454-1493) -, " qui jusqu'alors avait eu à Florence peu de succès, précisément à cause de l'influence de l'école néoplatonicienne " dont Ficin était le chef de file. "En plaçant son premier cours universitaire sous le signe de la philologie pure, étrangère, en somme, à la tradition de sa propre ville, il accomplit un acte novateur [...] qu'il est difficile de séparer du cadre de la politique culturelle alors mise en œuvre " par Laurent le Magnifique, "ce qui semble indiquer que, dès 1480, on commençait à considérer l'expérience ficinienne comme périmée "22.

Il était alors d'usage de commencer le cours par une leçon inaugurale (prælectio), morceau de bravoure rhétorique lu devant un public plus vaste que celui des étudiants. Le professeur y faisait l'éloge de la discipline enseignée et présentait l'auteur et l'œuvre qu'il allait commenter, conformément aux règles scolastiques de l'accessus ad auctores. La leçon inaugurale était la seule partie du cours véritablement faite pour être conservée ; nous possédons ainsi de nombreuses prælectiones des collègues de Politien ${ }^{23}$. En ce qui le concerne, non seulement la plupart de ses leçons inaugurales nous ont été transmises, mais, à partir de 1482, il les conçoit - du moins pour les plus importantes d'entre

21 Cette lettre figure dans toutes les éditions des œuvres de Pic (par exemple Opera omnia Joannis Pici, Bâle, Henricpetri, 1572, p. 348-351) ; on peut la lire en français dans : Jean Pic de la Mirandole, OEuvres philosophiques, trad. Olivier Boulnois, Paris, Presses universitaires de France, 1993 , p. 267-272.

22 Lucia Cesarini Martinelli, "Poliziano e Stazio : un commento umanistico " (précédemment publié sous le titre "In margine al commento di Angelo Poliziano alle Selve di Stazio ", Interpres, I, 1978), rééd. dans l'ouvrage collectif Il Poliziano latino, Galatina, Congedo, 1996, p. 59-102 (p. 74).

23 Præfationes Johanni Argyropuli dum Florentiæ doceret philosophiam (voir ci-dessus, note 7) ; Cristoforo Landino, Scritti critici e teorici, éd. Roberto Cardini, Rome, Bulzoni, 1974 ; V. R. Giustiniani, "L'orazione di Lorenzo Lippi per l'apertura dell'università di Pisa ", Rinascimento, IV, 1964, p. 265-284 ; Charles Trinkaus, "A humanist image of humanism : the inaugural orations of Bartolommeo della Fonte ", Studies in the Renaissance, VII, 1960, p. 90-147 ; Simona Mercuri, "Oratio in laudem oratoriæ facultatis di Bartolomeo Fonzio : testo e commento ", Interpres, XXIII, 2004, p. 54-84 ; Anna Meschini, "La prolusione fiorentina di Giano Làskaris ", in Miscellanea di studi in onore di Vittore Branca, t. III : Umanesimo e Rinascimento, Florence, Olschki, 1983, vol. 1, p. 69-113. 
elles - comme des œuvres littéraires à part entière, ayant vocation à figurer dans ses œuvres complètes ${ }^{24}$.

Le reste du cours, sauf lorsque l'auteur en décidait autrement, n'était pas destiné à la publication. Divers manuscrits contenant des notes préparatoires aux cours de Politien ont été conservés ${ }^{25}$, ainsi que des notes d'élèves prises dans les marges d'éditions imprimées ${ }^{26}$. Ces documents permettent de voir sur le vif la façon dont Politien travaillait. L'un des plus soignés est celui correspondant à son premier cours, sur les Silves de Stace ${ }^{27}$. Très copieux (752 pages dans l'édition critique), il se compose de notes tantôt autographes, tantôt mises au net par deux secrétaires. Le manuscrit s'ouvre sur " une partie introductive concernant la biographie de l'auteur, le genre littéraire et les circonstances de la découverte des Silves par Poggio Bracciolini "28 ; puis vient le commentaire proprement dit, sous la forme d'une longue série de lemmes suivant l'ordre du texte et expliquant de façon plus ou moins détaillée, parfois sur plusieurs pages, des mots ou des expressions rares ou difficiles, qui abondent dans l'œuvre de Stace. Le commentaire est en grande partie rédigé, manifestement prêt pour être lu. Les notes contiennent souvent des listes de citations parallèles, indiquant comment d'autres auteurs antiques avaient employé les mêmes mots. Politien ne considère pas ce commentaire comme véritablement achevé, puisqu'il l'a intitulé Tumultuaria commentatio, c'est-à-dire " commentaire hâtif " ou "désordonné ".

L'approche philologique radicale défendue par Ange Politien consiste non seulement à expliquer le sens des mots aux auditeurs, mais aussi à rétablir le texte sous sa forme la plus correcte possible. Il est intéressant de remarquer à ce propos que si, dans ses cours, Politien lisait directement l'œuvre de l'auteur commenté - de préférence à partir d'une édition imprimée -, il s'appuyait toujours, pour ses propres explications, sur un commentaire antérieur, qui lui servait en quelque sorte de matière première et qu'il passait au crible. Pour ce qui est de Quintilien, nous ne disposons que des annotations de Politien portées sur son exemplaire personnel d'une édition de l'Institutio oratoria imprimée à

24 Leur titre est indiqué, en annexe du présent article, à la suite du programme de chaque année scolaire.

La plupart ont fait l'objet d'éditions critiques, qui seront mentionnées au fil de cet article. Voir ci-dessous, note 63. 
Milan en $1476^{29}$. Dans le cas des Silves de Stace, il s'est servi du commentaire de l'humaniste Domizio Calderini (1443-1478) ${ }^{30}$, et il le critique pratiquement à chaque page. Comme le souligne Lucia Cesarini Martinelli ${ }^{31}$, Calderini était un humaniste plus " moderne " que le vieux Landino, et " polémiquer contre Calderini signifiait en un certain sens en recueillir l'héritage, tout en se présentant comme candidat à sa succession " au titre de chef de file de l'humanisme le plus avant-gardiste.

Tout en s'appuyant sur des commentaires antérieurs, Ange Politien - à l'instar de Barbaro, savant exégète de Pline l'Ancien - n'a pas de mots assez durs pour fustiger les « barbares " (c'est-à-dire les scolastiques) qui se contentent de commenter des commentaires, incapables qu'ils sont d'aborder directement les textes. Il n'y a pas là de contradiction, car précisément, chez Politien, l'analyse des commentaires préexistants constitue la première étape d'une restitution philologique du texte mais ne se substitue pas à ce dernier : le texte mis au programme est directement lu devant les élèves, et le professeur n'omet généralement pas de citer les auteurs qu'il critique. À la différence du cours magistral traditionnel, où le professeur tendait à substituer ses propres explications au texte étudié, la lectio telle que l'entend Politien est moins une "leçon " toute faite qu'une "lecture " en train de se faire.

\section{IV - Consolidation d'une position de prestige (1481-1490)}

Qui étaient les destinataires des cours d'Ange Politien ? Son enseignement universitaire comportait, d'une part, un grand " cours "officiel" consacré à un texte "important", qui commençait en novembre par une solennelle leçon inaugurale et se poursuivait vraisemblablement pendant toute l'année académique ", et d'autre part une série de " cours "complémentaires" qui pouvaient avoir lieu à divers moments de l'année et s'adressaient probablement à des groupes homogènes d'étudiants $"{ }^{32}$ - des sortes de séminaires de recherche, en somme. Une fois cette distinction posée, toutefois, il est assez difficile de

29 Florence, Biblioteca nazionale centrale, Banco rari 379 ; voir Alessandro Danieloni, Poliziano e il testo dell'Institutio oratoria, Messine, Centro interdipartimentale di studi umanistici, 2001.

30 Inclus dans l'édition des Silves (Silvarum libri V) publiée à Rome en 1475.

31 "Poliziano e Stazio : un commento umanistico ", op. cit., p. 66 et 73.

32 Lucia Cesarini Martinelli, "Poliziano professore allo Studio fiorentino ", op. cit., p. 479. 
différencier concrètement ces deux sortes de cours d'après les documents dont nous disposons.

En ce qui concerne le latin, le cours initial sur les Silves, d'un très haut niveau philologique, n'est pas tout à fait représentatif de l'ensemble de son enseignement. Cela s'expliquerait par "le caractère d'investiture que les premières leçons professées ex cathedra revêtaient pour le jeune maître "33. En se fondant sur les recueils de notes préparatoires qui ont été conservés, Cesarini Martinelli distingue deux sortes de cours : ceux sur les Silves (1480-81), sur les Fastes d'Ovide (1481-82) ${ }^{34}$ et sur les Satires de Perse (1484-85) ${ }^{35}$ paraissent destinés à un public " non pas de débutants, mais d'étudiants déjà avancés " ${ }^{36}$, tandis que les cours sur les Bucoliques de Virgile (1482-83) ${ }^{37}$ et sur l'Andria de Térence (1484-85) ${ }^{38}$ sont plus élémentaires, comme l'atteste le fait que Politien " se contente la plupart du temps de renvoyer au lexique latin de Giuniano Maio " 39 .

Les textes d'Ovide et de Perse sont plus difficiles que ceux de Virgile et de Térence, ce qui laisse effectivement supposer que Politien commentait les premiers devant des étudiants plus avancés que ceux auxquels étaient destinés les cours à caractère "didactique ". Mais le jugement de Cesarini Martinelli, qui estime que Politien s'adressait, dans ce type de cours, à " des débutants auxquels il fallait enseigner les premières règles grammaticales et les rudiments du lexique $"^{40}$, est sans doute excessif, puisque l'initiation à la langue latine était déjà assurée par les grammatici. Quoi qu'il en soit, Politien utilise pour son enseignement deux dictionnaires imprimés : celui de Giuniano Maio ${ }^{41}$ et celui de Giovanni Tortelli ${ }^{42}$. Il n'a pas beaucoup d'estime pour ces ouvrages, dont il

33 Lucia Cesarini Martinelli, "Poliziano e Stazio : un commento umanistico ", op. cit., p. 76.

34 Munich, Bayerische Staatsbibliothek, ms. Clm 754 ; Angelo Poliziano, Commento inedito ai Fasti di Ovidio, éd. Francesco Lo Monaco, Florence, Olschki, 1991.

35 Même manuscrit ; Angelo Poliziano, Commento inedito alle Satire di Persio, éd. Lucia Cesarini Martinelli et Roberto Ricciardi, Florence, Olschki, 1985.

36 Lucia Cesarini Martinelli, ibid., p. XVII.

37 Munich, Bayerische Staatsbibliothek, ms. Clm 754.

38 Même manuscrit; Angelo Poliziano, La commedia antica e l'Andria di Terenzio, éd. Rosetta Lattanzi Roselli, Florence, Sansoni, 1971.

39 Lucia Cesarini Martinelli, introduction au Commento inedito alle Satire di Persio, op. cit., p. XVII.

40 Lucia Cesarini Martinelli, " Poliziano e Stazio : un commento umanistico ", op. cit., p. 76.

41 Juniani Maii Parthenopei ... de priscorum proprietate verborum, Naples, 1475 (nombreuses rééditions).

42 Commentariorum grammaticorum de orthographia dictionum e Græcis tractorum opus, Rome, 1471 (nombreuses rééditions). 
relève souvent les insuffisances ; mais le fait même qu'il s'y réfère montre qu'ils faisaient partie des instruments de travail habituels des étudiants.

Dans le domaine du grec, à ses débuts, Ange Politien devait contractuellement fournir deux prestations ${ }^{43}$ : un cours ordinaire "en langue grecque " (in græco) - par quoi il faut entendre non pas un cours prononcé en grec mais un cours sur des textes grecs, "soit de poétique, soit de rhétorique " - et, à son domicile, des séances d'initiation à la pratique de la langue elle-même ${ }^{44}$. Cette dernière obligation cesse dès 1483 , date à laquelle il commence à mettre des auteurs grecs au programme de ses leçons publiques - Hésiode (1483-84), puis Homère (de 1485 à 1490) ${ }^{45}$-, ce qui signifie qu'il n'estimait pas disposer jusqu'alors d'un auditoire suffisamment aguerri pour que la première prestation prévue par son contrat pût être assurée. Son public, néanmoins, est partiellement composé de non-hellénistes, comme l'indique le passage de la leçon inaugurale sur l'Odyssée où il indique que ses commentaires s'adressent " aussi bien à ceux qui étudient la littérature grecque qu'à ceux qui se limitent aux lettres latines " 46 ; de fait, certains élèves " suivaient sur une version latine le texte grec commenté durant la leçon ${ }^{47}$. Au milieu de la décennie, Politien passe pour être meilleur helléniste que les Byzantins émigrés ; il ne manque pas de faire savoir tout le mal qu'il pense de son collègue et ancien professeur Argyropoulos, dont les auditeurs désertent les cours au profit des siens.

En dehors du cadre universitaire, Politien donne également des leçons privées à des élèves fortunés, tels que des fils de diplomates. En 1489-90, il commente ainsi l'Histoire naturelle de Pline et, l'année suivante, la Physique d'Aristote.

43 Voir ci-dessus, note 13.

44 Sur les cours de grec donnés par Politien, voir : Léon Dorez, "L'hellénisme d'Ange Politien ", Mélanges d'archéologie et d'histoire, XV, 1895, p. 3-32 ; Lucia Cesarini Martinelli, "Grammatiche greche e bizantine nello scrittoio del Poliziano ", dans l'ouvrage collectif Dotti bizantini e libri greci nell'Italia del secolo XV, Naples, D'Auria, 1992, p. 257-290.

45 Les notes préparatoires aux cours sur Homère sont conservées dans le ms. grec 3069 de la Bibliothèque nationale de France ; Angelo Poliziano, Appunti per un corso sull'Odissea : editio princeps dal ms. Par. gr. 3069, éd. Luigi Silvano, Alessandria, Edizioni Dell'Orso, à paraître. Voir également : Luigi Silvano, "Per la cronologia delle lezioni del Poliziano sull'Odissea ", Medioevo greco, I, 2001, p. 227-231 ; Id., "Angelo Poliziano : prolusione a un corso sull'Odissea ", Medioevo greco, II, 2002, p. 241-259. La leçon inaugurale de 1486-87 sur Homère vient de faire l'objet d'une édition critique : Angelo Poliziano, Oratio in expositione Homeri, éd. Paola Megna, Rome, Edizioni di storia e letteratura, 2007.

46 ... ut non his dumtaxat, qui græcam litteraturam affectant, sed et his pariter consulatur lenarratio mea], qui sunt latinitate contenti: Prælectio in enarrationem Odysseæ, 1489, éd. par Luigi Silvano, "Angelo Poliziano : prolusione a un corso sull'Odissea ", art. cit., p. 257.

Luigi Silvano, ibid., p. 251-252. 
Au fil de la décennie, l'aspect des notes préparatoires de Politien se modifie. Alors que le manuscrit du cours sur les Silves se présentait comme un commentaire, sinon achevé, du moins très élaboré, celui du cours sur les Fastes est un mélange de passages rédigés et de brouillons " chaotiques " ${ }^{48}$. Politien lui-même, d'ailleurs, ne le qualifie pas de "commentaire " mais de " notes recueillies en vue [ou à l'occasion] du commentaire des Fastes " (Collectanea in enarrationem Fastorum). Il s'agit en fait d'un recueil à usage personnel de " citations, passages parallèles et auctoritates sur des questions dont le texte des Fastes constitue la trame, le squelette ", si bien que "sur certains points les citations sont invraisemblablement longues et sortent manifestement des limites de l'exégèse, qui [...] apparaît comme un simple prétexte " ${ }^{49}$. Il est donc impossible de reconstituer, à partir de ces notes, le cours proprement dit.

La situation est la même en ce qui concerne le cours sur les Satires de Perse. Là aussi, nous avons affaire à un " brouillon " pouvant " susciter chez un lecteur moderne le doute et la perplexité ", étant donné que Politien s'y montre " particulièrement avare d'explications littérales susceptibles d'aider à s'orienter dans le texte, fort difficile, de Perse "; il est par conséquent " probable que certains éclaircissements aient été apportés oralement ", et l'éditrice du manuscrit s'avoue " dans l'incapacité, sur plus d'un point, de reconstituer avec exactitude la solution proposée par l'humaniste ", d'autant plus que, comme dans le cas des notes sur les Fastes, " on pourrait lui reprocher une certaine surabondance, un déploiement d'érudition parfois hors de proportion avec l'exégèse du texte " ${ }^{50}$. En outre, Politien avait mis au point tout un système de signes et de renvois d'un manuscrit à l'autre afin de ne pas avoir à recopier des citations en entier. Ainsi, ses notes deviennent de plus en plus brèves et elliptiques au fil des ans.

Indépendamment des besoins de ses étudiants et de leurs niveaux respectifs, Ange Politien poursuit, durant la première décennie de son enseignement, une efficace stratégie d'autopromotion. $\mathrm{Si}$, au début de sa carrière, il se démarque de ses collègues en choisissant des textes plus recherchés et difficiles que ceux de Cicéron ou de Virgile, il pense dès 1482 avoir suffisamment consolidé sa position pour pouvoir se permettre de donner, jusqu'en 1487, des cours sur

48 Francesco Lo Monaco, introduction au Commento inedito ai Fasti di Ovidio, op. cit., p. XVIII.

49 Ibid., p. XIX.

50 Lucia Cesarini Martinelli, introduction au Commento inedito alle Satire di Persio, op. cit., p. XVIXVII. 
Virgile $^{51}$ (et peut-être aussi sur Cicéron ${ }^{52}$ ) sans pour autant renoncer à sa personnalité propre. Et c'est précisément lorsqu'il s'empare de ce grand classique de l'enseignement universitaire qu'il invente un nouveau genre littéraire : la leçon inaugurale en vers. Ce sont les fameuses Silves ${ }^{53}$, ainsi intitulées parce que la silve antique était une poésie de circonstance, composée - en principe, du moins - dans le feu de l'action. Il y en aura quatre en tout : Manto (1482), sur les Bucoliques ; Rusticus (1483), sur les Géorgiques et Les Travaux et les Jours d'Hésiode ; Ambra (1485), sur Homère ; Nutricia (1486), sur la poésie en général. Politien poussera même l'originalité jusqu'à commenter ses propres silves, en 1486-87 et 1490-91. L'autocommentaire était une chose qui ne se faisait absolument pas, mais Ange Politien pouvait se le permettre. Il existe également un commentaire manuscrit sur la silve Ambra rédigé par Pietro Petreio, professeur à Rome, qui avait connu Politien à Florence dans les années 1490, et qui s'est probablement servi de l'autocommentaire du grand philologue (aujourd'hui perdu) pour composer le sien ${ }^{54}$.

Une nombreuse assistance se pressait désormais aux cours de Politien ; ceux de ses collègues se vidaient. Les mauvaises langues ne manquèrent pas pour dire qu'on venait voir les cours de Politien par snobisme et que la plupart des auditeurs ne comprenaient pas un mot de ce qu'il disait - ce dont apparemment il s'amusait lui-même, si l'on en croit une de ses leçons inaugurales ${ }^{55}$.

51 Le ms. Clm 754 de Munich contient des notes sur les Bucoliques (encore inédites) et les Géorgiques (éd. Massimo Gioseffi, "Angelo Poliziano e le postille pseudo-probiane a Virgilio ", Rendiconti dell'Istituto lombardo di scienze e lettere, CXXVI, 1992, p. 65-86), ainsi que sur le De rosis pseudo-virgilien (éd. Manlio Pastore Stocchi, "Il commento del Poliziano al carme De rosis ", in Miscellanea di studi in onore di Vittore Branca, op. cit., t. III, vol. 1, p. 397-422). Politien avait annoté, avant de devenir professeur, un exemplaire des Géorgiques imprimé à Rome en 1471 (Paris, BnF, Rés. g. Yc. 236 ; Angelo Poliziano, Commento inedito alle Georgiche di Virgilio, éd. Livia Castano Musicò, Florence, Olschki, 1990).

52 Politien est réputé avoir fait un cours sur l'Orator, impossible à dater (voir Lucia Cesarini Martinelli, "Poliziano professore allo Studio fiorentino ", op. cit., p. 479-480). En outre, des notes sur les Philippiques, attribuées à Ange Politien, sont conservées dans le ms. Clm 755 de la Bayerische Staatsbibliothek ; voir Ida Maïer, Les Manuscrits d'Ange Politien, Genève, Droz, 1965, p. 208. Le cours sur les Tusculanes, donné en 1492-1493, relève d'un autre contexte, s'agissant d'un écrit philosophique (voir ci-dessous, fin de la $5^{\mathrm{e}}$ partie).

53 Ange Politien, Les Silves, trad. Perrine Galand avec fac-similé des éditions originales, Paris, Les Belles Lettres, 1987 ; Angelo Poliziano, Silvæ, éd. Francesco Bausi, Florence, Olschki, 1997 ; Angelo Poliziano, Silvæ, éd. et trad. Charles Fantazzi, Cambridge (Mass.), Harvard University Press, 2004.

54 Alessandro Perosa, Un commento inedito all'Ambra di Poliziano, Rome, Bulzoni, 1994.

55 "De grâce, que personne ne se trouble si j'emploie parfois les termes mêmes des arts, et aussi des mots grecs puisque, pour la plupart, c'est ainsi qu'on les trouve écrits, car ils n'ont pas encore appris à parler latin ", Panepistemon, 1490-91. 
Ange Politien était enclin à la polémique, même lorsque ses adversaires n'étaient pas à sa mesure. Il discrédita en 1483 un autre professeur de poétique et rhétorique, Bartolomeo della Fonte (1445-1513). Celui-ci l'accusait d'avoir plagié son commentaire sur Perse ${ }^{56}$; sa situation étant devenue intenable, il démissionna de l'université de Florence ${ }^{57}$ avant d'être réintégré l'année suivante. C'était un modeste enseignant par rapport au grand Politien : il ne gagnait que 40 florins en 1481-82 (moins du tiers du salaire de Politien) ; mais il bénéficia d'une vengeance posthume puisque, après la mort de son détracteur en 1494, son salaire passa à 120 florins (1496-97), puis à 200 (1498-99).

L'arrogance de Politien lui valut bien d'autres inimitiés. Il fit aussi des envieux : par exemple, dans le manuscrit contenant les notes préparatoires au cours sur les Silves de Stace, "le nom de l'auteur [Ange Politien] a été recouvert d'une tache d'encre par Marcello Adriani, élève et successeur de Politien à l'université de Florence, qui a mis son propre nom à la place "58. En revanche, Pietro Crinito (Pietro Del Riccio, 1475-1507), héritier d'une partie des manuscrits d'Ange Politien, dont il avait été le disciple, fit relier ensemble les notes préparatoires aux cours sur Ovide ${ }^{59}$, Virgile, Perse, Térence et Suétone ${ }^{60}$ - tantôt autographes, tantôt copiées par ses secrétaires ou certains de ses élèves -, ce qui leur a permis de parvenir jusqu'à nous ${ }^{61}$; on lui doit également la survie du commentaire de la silve Ambra mentionné plus haut.

Enfin, des notes prises par des auditeurs d'Ange Politien à l'occasion des cours sur les Satires de Juvénal (1485-86) et sur l'Énéide de Virgile (1486-87) ont été conservées ${ }^{62}$, de même qu'un exemplaire des Silves de Stace

56 Bartholomei Fontii in Persium poetam explanatio, Florence, 1477.

57 Les registres portent pour l'année 1483-84 la mention "renuntiavit " : Armando Verde, Lo Studio fiorentino, op. cit., vol. I, p. 85.

58 Lucia Cesarini Martinelli, introduction au Commento inedito alle Selve di Stazio, op. cit., p. XIII.

59 En plus des notes consacrées aux Fastes, ce recueil contient des notes sur l'une des Héroïdes d'Ovide, correspondant à un cours de l'année 1480-1481 ; Angelo Poliziano, Commento inedito all'epistola ovidiana di Saffo a Faone, éd. Elisabetta Lazzeri, Florence, Sansoni, 1971.

60 Vincenzo Fera, Una ignota Expositio Suetoni del Poliziano, Messine, Centro di studi umanistici, 1983. Politien avait donné un cours en 1490-91 sur les Vies des douze Césars ; cf. Gianna Gardenal, Il Poliziano e Svetonio : contributo alla storia della filologia umanistica, Florence, Olschki, 1975.

61 C'est le manuscrit latin 754 de Munich, déjà cité à plusieurs reprises. Voir sa description dans Ida Maïer, Les Manuscrits d'Ange Politien, op. cit., p. 205-207.

62 Ravenne, Biblioteca Classense, ms. 237. 
(Rome, 1475) annoté par son élève Jacopo Salviati, qui avait suivi son cours en $1480-1481^{63}$.

\section{V - Le grammairien omniscient (1490-1494)}

Soucieux d'innover et de se renouveler sans cesse, Ange Politien se montre résolument moderne par sa façon d'utiliser ce qui est encore à l'époque une nouvelle technologie : l'imprimerie ${ }^{64}$. L'emploi, dans un cadre scolaire, d'éditions imprimées de textes classiques et de dictionnaires n'était pas en soi une chose extraordinaire ${ }^{65}$. En revanche, l'idée de confier à un imprimeur, presque immédiatement, ses propres leçons inaugurales les plus originales et les plus importantes est très significative, car elle traduit la volonté de marquer les esprits en rendant ces textes rapidement accessibles ${ }^{66}$. Cela contribue à faire de Politien un professeur à la pointe du progrès.

Ces initiatives s'inscrivent, une fois de plus, dans une politique culturelle cohérente. Ainsi, lorsque, en 1485, Politien préface l'editio princeps du De re ædificatoria, le premier traité d'architecture écrit depuis l'Antiquité, œuvre du Florentin Leon Battista Alberti (1404-1472), il dédie l'ouvrage à Laurent de Médicis, promoteur et mécène de la modernité florentine.

C'est dans un tel contexte que Politien fait paraître, en octobre 1489, un ouvrage qui est à la fois un bilan de ses dix premières années d'enseignement et l'annonce d'une nouvelle orientation : la Miscellaneorum centuria prima ${ }^{67}$, recueil de cent chapitres consacrés à des questions variées de lexicographie latine et grecque. Il y énonce sa conception de la philologie comme activité d’interprétation des textes nourrie de toutes les connaissances possibles : qui

63 Florence, Biblioteca Riccardiana, inc. Ed. R.369 ; cf. Lucia Cesarini Martinelli, " Poliziano e Stazio : un commento umanistico ", op. cit., p. 71.

64 Cette invention venue d'Allemagne avait été introduite en Italie dans les années 1460.

65 Landino se référait déjà dans ses cours, au temps où Politien était son élève, au De orthographia de Tortelli.

66 Ces six leçons inaugurales sont toutes imprimées à Florence chez Antonio Miscomini (sauf la silve Ambra, chez Niccolò di Lorenzo). Trois des quatre prælectiones en vers - les Silves - paraissent aussitôt après avoir été lues : Manto en novembre 1482, Rusticus en octobre 1483, Ambra en novembre 1485 ; il en va de même pour les deux prælectiones en prose, dont il sera question plus loin, qui marquent le tournant philosophique de Politien : Panepistemon paraît en février 1491, Lamia en novembre 1492. Seule la silve Nutricia (1486) est publiée à quelques années de distance, en mai 1491.

67 Angeli Politiani miscellaneorum centuria prima, Florence, Antonio Miscomini, 1489. 
veut interpréter les œuvres des anciens doit avoir parcouru l'" encyclie " tout entière, c'est-à-dire posséder une culture universelle ${ }^{68}$.

L'année suivante, Ange Politien franchit un nouveau pas en commençant à donner des cours sur l'œuvre d'Aristote. C'était une chose en principe interdite à un professeur de poétique et rhétorique - à l'exception des professeurs byzantins tels qu'Argyropoulos, pour d'évidentes raisons de maîtrise de la langue ; mais Politien pouvait se mesurer en la matière aux Grecs eux-mêmes, et il avait obtenu en 1488 le privilège de pouvoir enseigner " dans les disciplines de son choix " "69. Sa leçon inaugurale sur l'Éthique à Nicomaque, lue en novembre 1490 et imprimée en février 1491, qui aura un grand retentissement, est une prouesse lexicographique en forme d'aide-mémoire, sorte d'encyclopédie miniature où sont présentées les différentes branches de la connaissance et de l'action humaines, avec toutes leurs subdivisions. Proclamant qu'il entreprend une chose que nul n'a faite avant lui et poussant à son terme le principe énoncé dans ses Miscellanées, Politien annexe à la philologie toutes les disciplines ; et il choisit, de façon provocatrice, d'intituler son discours Panepistemon, c'està-dire $L^{\prime} O m n i s c i e n t^{70}$.

La réaction ne se fait pas attendre : les professeurs de philosophie dénoncent les prétentions de ce "grammairien " qui joue au philosophe et ose commenter les textes d'Aristote sans avoir reçu la formation adéquate. Mais Politien persiste et signe. Il fait savoir qu'il compte commenter l'intégralité du corpus aristotélicien, en commençant par l'Organon, qui figure au programme de ses cours à partir de $1491^{71}$. Et il empiète encore un peu plus sur les platesbandes de ses sourcilleux collègues en faisant précéder ses cours sur l'œuvre dialectique d'Aristote par l'examen de deux textes philosophiques à caractère propédeutique : l'Isagoge de Porphyre, philosophe du III ${ }^{e}$ siècle après J.-C., et le De sex principiis de Gilbert de la Porrée, auteur du XII siècle. L'utilisation de l'ouvrage du "Porrétain ", ainsi que celle des œuvres d'Hugues de Saint-

68 Ibid., ch. IV.

69 Voir ci-dessus, note 16.

70 J'ai donné en 1998, dans ma thèse de doctorat, une édition commentée de ce texte, à paraître aux éditions Droz. Pour une présentation rapide de la question, voir Jean-Marc Mandosio, "Filosofia, arti e scienze : l'enciclopedismo di Angelo Poliziano ", dans l'ouvrage collectif Poliziano nel suo tempo, Florence, Franco Cesati, 1996, p. 135-165.

71 Sur l'enseignement d'Ange Politien concernant la logique, voir Cesare Vasoli, "Il Poliziano maestro di dialettica " (1954), rééd. dans La dialettica e la retorica dell'umanesimo : invenzione e metodo nella cultura del XV e XVI secolo, Milan, Feltrinelli, 1968. 
Victor (autre théologien du XII ${ }^{\mathrm{e}}$ siècle) ${ }^{72}$, montre que Politien ne méprise pas systématiquement les commentateurs médiévaux. Le latin de Gilbert de la Porrée et d'Hugues de Saint-Victor est antérieur à la création de la terminologie scolastique, influencée par les traductions arabo-latines du corpus aristotélicien, et ne se confond pas à ses yeux avec l'horrible jargon des "modernes " tels que les occamistes ou les scotistes, qui l'horripile. Il apprécie également les commentaires d'Averroès et de Thomas d'Aquin.

Profitant de l'occasion pour faire preuve une nouvelle fois d'originalité, il consacre l'intégralité de sa leçon inaugurale de 1492, intitulée Lamia ${ }^{73}$, à sa polémique contre les philosophes professionnels. Dans ce texte mordant, il revendique pour lui-même le qualificatif, habituellement péjoratif, de grammaticus, en précisant qu'il n'entend nullement se faire passer pour un philosophe (cette prétention étant, selon lui, l'indice le plus sûr qu'on n'en est pas un) ; il laisse toutefois entendre que, malgré l'apparente modestie de sa tâche, le grammairien-philologue est en réalité supérieur à la plupart des prétendus philosophes, puisqu'il a les moyens, non seulement de lire Aristote et les autres philosophes antiques dans le texte original, mais de rétablir ce texte dans sa pureté lorsqu'il a été corrompu au lieu de se contenter, comme le tout-venant des professeurs de philosophie, de recourir à des éditions défectueuses et à des commentaires "barbares".

Il a été relevé que cette leçon inaugurale, lue à la rentrée 1492 et publiée dès le mois de novembre, est immédiatement postérieure à la mort de Laurent de Médicis, survenue en avril : " ce n'est qu'à ce moment-là que Politien, après trois ans de leçons sur des textes philosophiques, éprouve le besoin de se défendre ", comme s'il était devenu " plus vulnérable " après la disparition de son puissant protecteur ${ }^{74}$. Les ennemis de Politien ont effectivement dû redoubler d'ardeur, en essayant de l'isoler pour l'affaiblir ; mais il ne faut pas oublier que, dès 1490 , l'humaniste anticipait les critiques que sa nouvelle orientation philosophique ne manquerait pas de susciter ${ }^{75}$.

72 Voir Jean-Marc Mandosio, "Les sources antiques de la classification des sciences et des arts à la Renaissance ", dans l'ouvrage collectif Les Voies de la science grecque : études sur la transmission des textes de l'Antiquité au dix-neuvième siècle, Genève, Droz, 1997, p. 331-390 (p. 350-351).

73 Angelo Poliziano, Lamia : prælectio in priora Aristotelis Analytica, éd. Ari Wesseling, Leyde, Brill, 1986.

74 Lucia Cesarini Martinelli, "Poliziano professore allo Studio fiorentino ", op. cit., p. 481.

75 «Et il ne m'échappe pas [...] que ce que je promets là tombe à pic pour mes détracteurs. [...] Mais il ne faut tenir absolument aucun compte des détracteurs ; si l'occasion leur manque aujourd'hui, ils en trouveront facilement une autre ", Panepistemon, 1490-91. 
Toujours sur le front philosophique, Politien s'attaque en 1492-93 aux Tusculanes de Cicéron. Dans les Miscellanées, il avait durement polémiqué contre Argyropoulos, qui prétendait que seuls les Grecs pouvaient comprendre la philosophie et que les Latins - à commencer par Cicéron - ne faisaient qu'embrouiller les choses, par méconnaissance ${ }^{76}$. Le fait de commenter une œuvre philosophique de Cicéron après avoir remis en cause la compétence d'Argyropoulos montre que Politien entendait embrasser les traditions philosophiques grecque et latine, toutes deux également légitimes à ses yeux et, en dernière analyse, pleinement concordantes.

\section{VI - Une postérité en demi-teinte}

La réalisation de l'ambitieux programme d'Ange Politien est interrompue par sa mort soudaine, à l'âge de quarante ans, le 28 septembre $1494^{77}$. Mais déjà le climat intellectuel et politique avait commencé à changer avec la mort de Laurent le Magnifique en 1492, qui avait plongé Politien, inquiet pour son avenir, dans le désarroi ; le fils de Laurent, Pierre, se révéla faible et indécis, incapable de maintenir les équilibres politiques précaires de l'Italie, et sera renversé à l'approche des troupes françaises, en novembre 1494. Barbaro, qui avait conçu le projet de retraduire tout Aristote, était mort en 1493 ; le grand ami de Politien, Pic de la Mirandole, mourra quelques semaines après lui, le 17 novembre 1494. Moins de dix ans plus tard, l'université de Florence cessera d'exister. Par une ironie de l'histoire, les anciennes gloires intellectuelles florentines, que Politien et Pic avaient voulu supplanter, leur survivront plusieurs années : Landino meurt en 1498, Ficin en 1499.

Après la mort d'Ange Politien, une partie de ses manuscrits est dispersée, malgré les efforts de son disciple Crinito pour rassembler les œuvres complètes du philologue, dont l'editio princeps voit le jour sous sa direction ${ }^{78}$. Ainsi, la Miscellaneorum centuria secunda, inachevée, sur laquelle Politien travaillait au moment de sa mort, ne sera retrouvée et publiée qu'au $\mathrm{XX}^{\mathrm{e}}$ siècle ${ }^{79}$.

76 Miscellaneorum centuria prima, op. cit., ch. I.

77 On s'est longtemps interrogé sur les causes de cette mort ; il semblerait qu'il ait été empoisonné (par un professeur de philosophie mécontent ?).

78 Omnia opera Angeli Politiani et alia quædam lectu digna, Venise, Alde Manuce, 1498.

79 Angelo Poliziano, Miscellaneorum centuria secunda, éd. Vittore Branca et Manlio Pastore Stocchi, Florence, Alinari, 1972. 
Par la suite, les philologues se nourriront de ses prælectiones et de sa Miscellaneorum centuria prima, constamment disponibles dans la première moitié du XVI ${ }^{\mathrm{e}}$ siècle, séparément ou au sein de ses œuvres complètes. Pas moins de quatre commentaires des Silves seront publiés - par Johann Murmelling en 1510, par Nicolas Bérault en $1513^{80}$, par Johann Ludwig Kohlbürger en 1548, et par Francisco Sanchez en 1554 -, ce qui donne une idée du succès de ces leçons inaugurales en vers. Le Panepistemon, pour sa part, servira de matrice à plusieurs projets encyclopédiques durant la même période ${ }^{81}$. La méthode philologique de Politien sera transmise par ses anciens élèves et, d'une façon beaucoup plus difficile à appréhender, par l'utilisation de ses notes manuscrites ${ }^{82}$. Ainsi, les cours de Politien exerceront, directement ou indirectement, une durable influence sur l'humanisme européen.

\author{
Jean-Marc MANDOSIO \\ École pratique des hautes études \\ jean-marc.mandosio@ephe.sorbonne.fr
}

80 Voir Perrine Galand-Hallyn, "Nicolas Bérault lecteur de Politien ", in Poliziano nel suo tempo, op. cit., p. 411-427 ; Nicolas Bérault, Leçon d'introduction et commentaire au Rusticus de Politien (éd. de 1518), éd. et trad. Perrine Galland-Hallyn, Genève, Droz, à paraitre.

81 Voir Jean-Marc Mandosio, "Filosofia, arti e scienze : l'enciclopedismo di Angelo Poliziano ", op. cit. ; Id., " La fortune du Panepistemon d'Ange Politien en France au XVI e siècle ", dans l'ouvrage collectif La Réception des écrits italiens en France à la Renaissance : ouvrages philosophiques, scientifiques et techniques, Paris, Centre interuniversitaire de recherche sur la Renaissance italienne, 2001, p. 49-71.

82 Pour ne prendre qu'un exemple, l'influence d'Ange Politien sur l'humaniste français Guillaume Budé (1468-1540), admise de fort mauvais gré par l'intéressé, est bien connue. Elle passe non seulement par les écrits publiés de Politien, que Budé a attentivement lus, mais aussi par sa rencontre en 1505 avec Crinito, qui lui donne accès aux annotations de Politien sur les Pandectes de Justinien (voir Luigi-Alberto Sanchi, " Dall'Italia alla Francia : l'erudizione di Guillaume Budé ", à paraître dans la revue Studi umanistici piceni en 2009). 


\section{Annexe}

\section{Chronologie des cours et des écrits didactiques d'Ange Politien ${ }^{83}$}

(Sont d'abord indiqués les textes qui ont fait l'objet du cours pendant l'année scolaire concernée, puis les écrits de Politien à la même période.)

$1480-81$

Quintilien, Institution oratoire ; Stace, Silves ; Ovide, Héroüdes (lettre de Sappho).

- Oratio super Fabio Quintiliano et Statii sylvis ; Commento inedito alle Selve di Stazio ; Commento inedito all'epistola ovidiana di Saffo a Faone.

1481-82

Ovide, Fastes ; ps.-Cicéron, Rhétorique à Herennius ; Erotemata (leçons privées).

- Commento inedito ai Fasti di Ovidio.

1482-83

Virgile, Bucoliques ; Erotemata (leçons privées).

- Manto (première " silve ", sur les Bucoliques de Virgile).

1483-84

Virgile, Géorgiques ; Hésiode, Les Travaux et les jours ; Ésope, Fables.

- Rusticus (deuxième " silve ", sur les Géorgiques de Virgile et Les Travaux et les jours d'Hésiode) ; Commento inedito alle Georgiche di Virgilio.

1484-85

Perse, Satires ; Horace, Satires ; ps.-Virgile : De rosis ; Térence, Andria ${ }^{84}$. - Praelectio in Persium; Commento inedito alle Satire di Persio ; La Commedia antica e l'Andria di Terenzio ; Il commento del Poliziano al carme De rosis.

1485-86

Homère, Iliade ; Juvénal, Satires.

- Ambra (troisième "silve ", sur Homère).

1486-87

Homère, Iliade ; Virgile, Énéide ; leçons sur la poésie en général ; commentaire de sa propre "silve " Ambra.

- Oratio in expositione Homeri ; Nutricia (quatrième " silve ", sur la poésie).

1487-88

Homère, Iliade; Virgile, Énéide.

83 Cette chronologie s'appuie principalement sur les données fournies par Lucia Cesarini Martinelli ("Poliziano professore allo Studio fiorentino ", op. cit.), complétées par Luigi Silvano (" Per la cronologia delle lezioni del Poliziano sull'Odissea ", op. cit.). Les cours de Politien sur Plaute et sur l'Orator de Cicéron n'ont pas pu être datés.

84 La datation de ces cours est conjecturale ; ils pourraient avoir été donnés ultérieurement. 
1488-89

Homère, Iliade ; Virgile, Énéide.

- Miscellaneorum centuria prima.

1489-90

Homère, Odyssée ; Pline l'Ancien, Histoire naturelle (leçons privées).

- Prælectio in enarrationem Odysseæ.

1490-91

Aristote, Éthique à Nicomaque et (sous forme de leçons privées) Physique ; Suétone, Vies des douze Césars ; commentaire de sa propre "silve " Nutricia.

- Praelectio cui titulus Panepistemon; Praefatio in Suetonii expositionem; Una ignota Expositio Suetonii del Poliziano.

\section{1-92}

Porphyre, Isagoge ; Gilbert de la Porrée, Les Six principes ; Aristote, Catégories, De l'Interprétation et Réfutations sophistiques ; Éthique à Nicomaque.

- Praelectio de dialectica.

1492-93

Aristote, Premiers analytiques ; Cicéron, Tusculanes.

- Lamia : praelectio in priora Aristotelis analytica; Dialectica.

1493-94

Aristote, Seconds analytiques et Topiques ; Ovide, Elégies (?).

- De Ovidii exilio et morte; Miscellaneorum centuria secunda (inachevée).

Ange Politien meurt le 28 septembre 1494. 